\title{
Nosocomial outbreak of KPC-2- and NDM-1-producing Klebsiella pneumoniae in a neonatal ward: a retrospective study
}

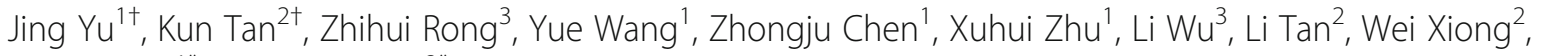
Ziyong Sun ${ }^{* *}$ and Ling Chen ${ }^{3 *}$

\begin{abstract}
Background: The spread of resistance to carbapenems among Enterobacteriaceae has become a major public health problem in recent years. In this study, we describe an outbreak of Klebsiella pneumoniae in the neonatal ward. First, we aimed to study the drug resistance, genetic relatedness, and transmission mechanism of carbapenem-resistant K. pneumoniae; second, we implemented infection control measures to contain the outbreak.

Methods: We investigated 27 non-repetitive strains isolated from neonates and five strains cultured from around the neonatal ward. Polymerase chain reaction (PCR), the agar dilution method, and multilocus sequence typing (MLST) were used to analyze the resistance gene(s), antimicrobial susceptibility, and homology, respectively. Health-care personnel education, hand hygiene, outer gown changing, and infected patient isolation were strictly enforced.

Results: Our antimicrobial susceptibility results show that all strains were multidrug-resistant. MLST and PCR results revealed that, in this study, all of the KPC-2-producing strains are Sequence Type (ST) 11 (ST11) $(n=22)$ and all of the NDM-1-producing strains are ST20 $(n=4)$ or ST888 $(n=1)$. The environmental strains were identified as KPC-2-positive K. pneumoniae ST11 $(n=3)$ and NDM-1-positive K. pneumoniae ST20 $(n=2)$. The percentages of isolates with the extended-spectrum- $\beta$-lactamases CTX-M-15, blaCTX-M-14, blaTEM-1 were 9.4, 84.3, and $68.8 \%$, respectively. AmpC $\beta$-lactamase genes were not detected in our isolates.

Conclusions: KPC-2-positive K. pneumoniae ST11 and NDM-1-positive K. pneumoniae ST20 were associated with this outbreak. The identification of these isolates in samples from radiant warmers and nurses suggests that hospital cross-transmission played a role in this outbreak. Active infection control measures were effective for controlling this multidrug-resistant K. pneumoniae outbreak.
\end{abstract}

Keywords: Multidrug-Resistant, Klebsiella pneumoniae, KPC-2, NDM-1, Nosocomial Infection, Neonate

\footnotetext{
*Correspondence: zysun@tjh.tjmu.edu; cntjszyong@163.com;

790356760@qq.com

${ }^{\dagger}$ Equal contributors

'Department of Clinical Laboratory, Tongji Hospital, Tongji Medical College Huazhong University of Science and Technology, Jiefang Road 1095\#, Wuhan 430000, China

${ }^{3}$ Department of Pediatrics, Tongji Hospital, Tongji Medical College, Huazhong

University of Science and Technology, Jiefang Road 1095\#, Wuhan 430000,

China

Full list of author information is available at the end of the article
} 


\section{Background}

Carbapenem-resistant Enterobacteriaceae (CRE) have increasingly been found to cause nosocomial infections during the past decade; they primarily affect critically ill patients [1]. Carbapenem-resistant Klebsiella pneumoniae (CR-KP) is one of the most important types of CRE, and it has spread extensively around the world [2]. Carbapenems are the most effective drug for treating infections caused by $K$. pneumoniae that produce extended-spectrum beta-lactamases (ESBLs). However, with the increase of carbapenem resistance in $K$. pneumoniae, options for treatment have become limited [3].

Carbapenemases, including KPC, GES, SME, NMC, IMI NDM, IMP, VIM, and OXA-48, are the main mechanisms of carbapenem resistance in CRE. Other mechanisms of carbapenem resistance in these bacteria include the absence or decreased expression of outer membrane proteins and mutations in the ampC enzyme or ESBLs [4].

Outbreaks of hospital-associated CRE infections are not rare $[5,6]$. In recent years, outbreaks of CRE infections in neonates have been rising and have attracted increasingly more attention [7, 8]. Here, we noted that the frequency of $K$. pneumoniae isolated from newborns in our neonatal ward had dramatically increased compared with that of previous years. Therefore, we collected CR-KP in 2015 from January to November to study the resistance and transmission mechanisms of carbapenem-resistant strains in the neonatal ward.

\section{Methods}

\section{Setting of the study}

A retrospective study was conducted in an 11months period between January 2015and November 2015 in the neonatal ward of Tongji Hospital (TJH). The neonatal ward of $\mathrm{TJH}$ receives approximately 1750 admissions per year and it has six rooms (60 beds) for newborns. The $60 \%$ of patients come from maternity ward and $30 \%$ of neonates were outpatients.

\section{Bacterial isolates collection and identification}

Thirty-two carbapenem-resistant $K$. pneumoniae were included in this study. Twenty-seven isolates were collected from different patients. At the same time, according to previous investigation, hospital infection control staff collected environmental samples from high frequency contact areas in neonatal ward in September 8, 2015, including bed railing, stethoscope, personal digital assistant, suction apparatus, venous catheter, radiant warmer, nurses' hand, thermostat button and ventilator machine. Samplings were accomplished before routine cleaning, 89 environmental samples and 40 samples from nurses' hands were obtained. All isolated strains were identified by conventional biochemical methods, then were confirmed by PCR amplification of $16 \mathrm{~S}$ rRNA gene and sequencing [9].

\section{PCR and DNA sequence analysis of resistance gene}

PCR were performed to detect drug-resistance genes, including carbapenemases (blaKPC-2, blaGES, blaIMP-4, blaVIM-1, blaNDM-1, blaOXA-48), common ESBL genes (blaCTX-group1, blaCTX-group2, blaCTX-group8, blaCTX-group9, blaTEM-1, and blaSHV), and ampC genes (MOX, FOX, DHA, CIT, AAC and EBC) [10-12]. The PCR products were sequenced and analyzed using BLAST (http://www.ncbi.nlm.nih.gov/BLAST).

\section{Susceptibility testing}

Antibiotic susceptibility testing was assayed by agar dilution method to determinate the minimum inhibitory concentration (MICs) of ampicillin, ceftazidime, cefotaxime, cefepime, cefuroxime, cefoxitin, gentamicin, levofloxacin, amikacin, ciprofloxacin, aztreonam, imipenem, meropenem, trimethoprim-sulfamethoxazole, piperacillintazobactam, tigecycline and colistin. All antibiotics, except tigecycline and colistin, were interpreted according to the standard of the Clinical and Laboratory Standards Institute (CLSI) document M100-S22. For tigecycline and colistin, the European Committee on Antimicrobial Susceptibility Testing (EUCAST) breakpoint was used. E. coli ATCC 25922 and P.aeruginosa ATCC27853 were used as quality control.

\section{Multilocus sequence typing (MLST)}

Epidemiological relatedness was carried out by multilocus sequence typing (MLST). Multilocus sequence typing (MLST) for clinical K. pneumoniae isolates was performed following the methods described previously [13]. The allelic profiles and sequence types (STs) were assigned using online databases (http://www.pasteur.fr/recherche/ genopole/PF8/mlst/Kpneumoniae.html).

\section{Infection control interventions}

Strict infection control measures were implemented according to the Guidance for Control of CRE in November, 2015 [14]. Healthcare personnel are familiar with proper hand hygiene technique and wash their hands properly before entering and upon leaving patient rooms. Hand hygiene adherence was monitored by video. Patients who are suspected or confirmed with CRE should be placed in a private room in which staff and visitors are required to don gloves and gowns for entry. Extensive cleaning of shared equipment and the use of disposable materials (when feasible) were implemented. 


\section{Results}

Epidemiological characteristics of $K$. pneumoniae isolates The molecular epidemiology of carbapenem-resistant Enterobacteriaceae was studied from January to November 2015. During this period, There were 1519 patients admitted to hospital,including 590 females (38.9 \%) and 929 males $(61.1 \%)$. The sex ratio of all patients is similar to infection group (61.1 vs $63.0 \%$ ). The average age of the CR-KP infection $(32.5 \pm 22.7)$ ws older than all patients $(11.2 \pm 23.4) .27$ consecutive CRE were isolated from 27 different patients. The characteristics of the CR-KP isolated in this study are shown in Table 1. Twenty seven of the isolates are from newborns in the neonatal unit, including 17 males and 10 females, with a mean age of 32.5 days (range, $1-85$ days). The sources of these specimens were blood $(n=9)$, urine $(n=8)$, aspiration catheter $(n=5)$, and sputum $(n=5)$. Meanwhile, ten suspicious colonies were found and further identified by biochemical method, and finally five CR-KP isolates were cultured from radiant warmers or nurses' hands during the peak of the outbreak.

Table 1 Characteristics of multi-drug resistance $K$. pneumoniae

\begin{tabular}{|c|c|c|c|c|c|c|}
\hline Isolate No. & sex & Specimen & Date Of detection & STs & Carbapenemase & ESBL \\
\hline TJ1 & $\mathrm{F}$ & Sp & 23/1/2015 & ST11 & $\mathrm{KPC}-2$ & CTX-14,TEM-1 \\
\hline TJ2 & M & $\mathrm{Bl}$ & $1 / 2 / 2015$ & ST888 & NDM-1 & CTX-15,TEM-1 \\
\hline TJ3 & M & $\mathrm{Ca}$ & 6/3/2015 & ST11 & KPC-2 & CTX-14,TEM-1 \\
\hline TJ4 & M & $\mathrm{Bl}$ & $7 / 3 / 2015$ & ST11 & $\mathrm{KPC}-2$ & CTX-15 \\
\hline TJ5 & M & Sp & 15/7/2015 & ST11 & KPC-2 & CTX-14,TEM-1 \\
\hline TJ6 & M & Sp & $16 / 7 / 2015$ & ST11 & $\mathrm{KPC}-2$ & CTX-14 \\
\hline TJ7 & $\mathrm{F}$ & Ur & $18 / 7 / 2015$ & ST20 & NDM-1 & TEM-1 \\
\hline TJ8 & M & $\mathrm{Ca}$ & 4/8/2015 & ST11 & $\mathrm{KPC}-2$ & CTX-14 \\
\hline TJ9 & M & $\mathrm{Ca}$ & 8/8/2015 & ST11 & $\mathrm{KPC}-2$ & CTX-14,TEM-1 \\
\hline TJ10 & $\mathrm{F}$ & $\mathrm{Bl}$ & $11 / 8 / 2015$ & ST11 & $\mathrm{KPC}-2$ & CTX-14,TEM-1 \\
\hline TJ11 & M & Ur & 26/8/2015 & ST11 & $\mathrm{KPC}-2$ & CTX-14,TEM-1 \\
\hline TJ12 & M & $\mathrm{Bl}$ & $31 / 8 / 2015$ & ST11 & KPC-2 & CTX-15 \\
\hline TJ13 & M & $\mathrm{Bl}$ & $4 / 9 / 2015$ & ST11 & $\mathrm{KPC}-2$ & CTX-14,TEM-1 \\
\hline TJ14 & $\mathrm{F}$ & $\mathrm{Ca}$ & 13/9/2015 & ST20 & NDM-1 & CTX-14,TEM-1 \\
\hline TJ15 & M & Sp & 13/9/2015 & ST11 & KPC-2 & CTX-14,TEM-1 \\
\hline TJ16 & M & $\mathrm{Ca}$ & 22/9/2015 & ST20 & NDM-1 & CTX-14 \\
\hline TJ17 & $\mathrm{F}$ & Sp & 25/9/2015 & ST11 & $\mathrm{KPC}-2$ & CTX-14,TEM-1 \\
\hline TJ18 & $\mathrm{F}$ & Ur & 30/9/2015 & ST11 & $\mathrm{KPC}-2$ & CTX-14,TEM-1 \\
\hline TJ19 & M & $\mathrm{Bl}$ & 2/10/2015 & ST11 & KPC-2 & CTX-14,TEM-1 \\
\hline TJ20 & $\mathrm{F}$ & Ur & $6 / 10 / 2015$ & ST11 & $\mathrm{KPC}-2$ & CTX-14,TEM-1 \\
\hline TJ21 & M & Ur & $8 / 10 / 2015$ & ST11 & $\mathrm{KPC}-2$ & CTX-14,TEM-1 \\
\hline TJ22 & $\mathrm{F}$ & Ur & $12 / 10 / 2015$ & ST11 & $\mathrm{KPC}-2$ & CTX-14,TEM-1 \\
\hline TJ23 & M & $\mathrm{Bl}$ & 25/10/2015 & ST11 & $\mathrm{KPC}-2$ & - \\
\hline TJ24 & M & $\mathrm{Bl}$ & 28/10/2015 & ST20 & NDM-1 & CTX-14 \\
\hline TJ25 & $\mathrm{F}$ & Ur & 20/11/2015 & ST11 & KPC-2 & CTX-14,TEM-1 \\
\hline TJ26 & $\mathrm{F}$ & $\mathrm{Bl}$ & 20/11/2015 & ST11 & KPC-2 & CTX-14 \\
\hline TJ27 & M & Ur & 21/11/2015 & ST11 & $\mathrm{KPC}-2$ & CTX-14,TEM-1 \\
\hline $\mathrm{TJ} 28^{\mathrm{a}}$ & - & Radiant warmer & 8/9/2015 & ST11 & KPC-2 & CTX-14,TEM-1 \\
\hline TJ29 & - & Radiant warmer & 8/9/2015 & ST20 & NDM-1 & CTX-14 \\
\hline $\mathrm{TJ} 30^{\mathrm{b}}$ & - & Nurses hand & 8/9/2015 & ST11 & KPC-2 & CTX-14,TEM-1 \\
\hline TJ31 ${ }^{c}$ & - & Radiant warmer & 8/9/2015 & ST11 & $\mathrm{KPC}-2$ & CTX-14,TEM-1 \\
\hline TJ32 ${ }^{\mathrm{b}}$ & - & Nurses hand & 8/9/2015 & ST2O & NDM-1 & CTX-14 \\
\hline
\end{tabular}

$F$ female, $M$ male, $S p$ sputum, $B L$ blood, Ur urine, $C a$ aspiration catheter ${ }^{\mathrm{a}} 65$ bed radiant warmer; ${ }^{\mathrm{b}}$ nurses hand; ${ }^{\mathrm{C}} 55$ bed radiant warmer 


\section{Antimicrobial susceptibility patterns of $K$. pneumoniae isolates}

The results of minimum inhibitory concentration (MIC) assays are shown in Table 2. All K. pneumoniae isolates were non-susceptible to ampicillin, cefoxitin, cefuroxime, cefotaxime, cefepime, ceftazidime, imipenem, meropenem, and piperacillin tazobactam, but were highly sensitive to tigecycline and colistin. The antibiotic resistance rates to gentamicin, amikacin, levofloxacin, ciprofloxacin, aztreonam, and trimethoprim-sulfamethoxazole were 78.1, 78.1, 78.1, 84.1, 78.1, and $21.9 \%$, respectively. Notably, strains producing NDM-1carbapenemase show different antibacterial susceptibility spectra from strains producing KPC-2 carbapenemase, including gentamicin, amikacin, levofloxacin, ciprofloxacin, aztreonam, and trimethoprim-sulfamethoxazole (see Table 2).

Sequence typing and expression of drug-resistance genes Based on the multi-locus sequence typing (MLST) results, the 27 isolates from unique patients were identified as Sequence Type (ST) 11 (ST11) $(n=22)$, ST20 $(n=4)$, and ST888 $(n=1)$, and the five environmental isolates were identified as ST11 $(n=3)$ and ST20 $(n=2)$.

Table 2 Antibiotic susceptibility of K. pneumoniae

\begin{tabular}{|c|c|c|}
\hline \multicolumn{2}{|c|}{ Minimal inhibitory concentration (ug/ml) } & \multirow{3}{*}{$\begin{array}{l}\text { NDM-1 positive K. pneumoniae } \\
(n=7) \\
\text { range }\end{array}$} \\
\hline Antibiotics & $\begin{array}{l}\text { K. pneumoniaeST11 } \\
(n=25)\end{array}$ & \\
\hline & range & \\
\hline AMP & $\geq 512$ & $\geq 512$ \\
\hline CAZ & $\geq 512$ & $\geq 512$ \\
\hline CTX & $128->512$ & 64- > 512 \\
\hline FOX & $64-256$ & $64-256$ \\
\hline FEP & $64-256$ & $16-64$ \\
\hline CXM & $\geq 512$ & $\geq 512$ \\
\hline ATM & $\geq 512$ & $1-2$ \\
\hline GN & $64-256$ & $0.25-0.5$ \\
\hline LEV & $32-128$ & $0.06-0.25$ \\
\hline AK & $\geq 512$ & $1-2$ \\
\hline CIP & $\geq 32$ & $0.06-1$ \\
\hline TZP & $64 / 4->256 / 4$ & $128 / 4->256 / 4$ \\
\hline SXT & $1 / 19-2 / 38$ & $\geq 256 / 4864$ \\
\hline IPM & $8-64$ & $1-16$ \\
\hline MEM & $4-32$ & $1-32$ \\
\hline TGC & $0.06-0.5$ & $0.06-0.5$ \\
\hline CO & $0.03-0.5$ & $0.06-0.25$ \\
\hline
\end{tabular}

AMP ampicillin (0.06-512), CAZ ceftazidime (0.06-512), CTX cefotaxime (0.06-512), FEP cefepime (0.06-512), CXM cefuroxime (0.06-512), FOX cefoxitin (0.06-512), GN gentamicin (0.06-512), LEV levofloxacin (0.06-512), AK amikacin (0.06-512), CIP ciprofloxacin (0.004-32), ATM Aztreonam (0.06-512), IPM imipenem (0.03-256), MEM meropenem (0.03-256), TZP piperacillin-tazobactam (0.25/4-256/4), SXT trimethoprim-sulfamethoxazole (0.25/4.75-256/4864), TGC tigecycline (0.03-256), CO colistin (0.03-256)
The percentages of isolates with carbapenemases KPC-2 and NDM-1were $81.5 \%(22 / 27)$ and $18.5 \%(5 / 27)$, respectively. All KPC-2-producing strains were ST11, and most of the NDM-1-positive strains were ST20 $(n=4)$. Three of the five environmental strains were KPC-2positive K. pneumoniae ST11, and the other two environmental isolates were NDM-1-positive $K$. pneumoniae ST20. The rates of the ESBLs CTX-M-15, CTX-M-14, and TEM-1 were 9.4, 84.3, and 68.8\%, respectively. AmpC genes and other carbapenemases were not detected.

\section{The effect of infection control interventions}

We monitored for neonatal infection with $K$. pneumoniae in future 4 months, and we detected multidrug-resistant $K$. pneumoniae in five patients, three patients, one patient and one patient, respectively, over these 4 months.

\section{Discussion}

K. pneumoniae is an important nosocomial pathogen that can cause pneumonia, sepsis, and meningitis [15]. The emergence of CR-KP has created a challenge for treating K. pneumoniae infections, the mortality caused by CR-KP incection was high [16]. However, one infected neonate has died in our study. Mortality from K. pneumoniae infection is associated with a variety of factors, including underlying disease, nutritional status, and medical treatment, which may explain the discrepancy in mortality among different studies.

Underlying disease, low birth weight, antibacterial drug treatment, invasive procedures, and prolonged hospitalization have all been reported as extremely important risk factors of CRE infection $[17,18]$. Of the 27 patients in this study, the percentages of subjects who had a low birth weight, who were treated with antibacterial drugs, and who underwent invasive procedures were $74.1 \%(n=20), 100 \%(n=27)$, and $77.8 \%(n=21)$ (data was not show), respectively. Additionally, we noticed that each nurse needed to take care of several infants and that each newborn was usually cared for by several nurses due to scheduling reasons. Overall, the newborns in our study possessed high risk factors for CRE infection, and this outbreak was likely spread by cross-infection.

As results showed, no significant differences in sex ratio and age between infection patients and non-infection patients. The CR-KP infection probably has no relation with age and gender in our study,which was similar with other study [19]. In addition, the average days for hospitalization in $\mathrm{TJH}$ is 11.8 days at present. However, the average hospital stay of the 27 patients including in this study was 29.9 days. Prolonged hospitalization may contribute to spreading of the ST11 and ST20 strains in neonatal ward. 
The first KPC-2-producing K. pneumoniae isolate (TJ1) was isolated from a 23-day-old boy that was confirmed as infected with multidrug-resistant $K$. pneumoniae at another hospital. He was transferred to our hospital for treatment on January 23, 2015, and a sputum culture confirmed his infection with a multidrug-resistant $K$. pneumoniae. We learned that his mother had suffered from a respiratory tract infection for 2 weeks and 4 days. Through combination therapy with cefoperazone sulbactam, amoxicillin clavulanic acid, fluconazole, and meropenem, the child recovered and was discharged on February 5, 2015. Subsequently, KPC-2-positive strains with the same phenotype appeared in the same ward. All KPC-2-positive strains were detected in patients who had been hospitalized for more than $48 \mathrm{~h}$, so we speculate that the origin of the KPC-2-positive $K$. pneumoniae ST11 was probably TJ1. Rodloff MD and his colleagues found that carriage of KPC-2-producing $K$. pneumoniae can last for 3 years [20]. It probably explain continuous outbreak after index case. Regrettably, the origin of the NDM-1-producing isolates in our study is still unknown.

The number of infection with $K$. pneumoniae decrease sharply in future 4 months. These declining numbers suggest that our efforts to control the continuous outbreak of multidrug-resistant $K$. pneumoniae were at least partially effective. However, there was one or two CR-KP detected after infection control interventions. It is difficult to eradicate the CR-KP by infection control measures [21]. Infection control measures were still need to improve, including routine screening of hospitalized patients, pathogens culture of medical equipment and screening the colonization status of contemporaneous patients.

This study has several limitations. Specifically, we only performed one environmental sampling and neglect screening of patients with perianal swabs to detect the rate of colonized patients. Additional samples might have provided stronger evidence to support our conclusion that the CR-KP was spread through cross-infection. Our data is also unable to confirm whether or not the CR-KP strains isolated in the later 3 months of our study are the same as the previous epidemic strain.

\section{Conclusions}

In summary, we report that KPC-2-positive $K$. pneumoniae ST11 and NDM-1-positive $K$. pneumoniae ST20 are the two main clones that caused neonate infection in our hospital during the study period. During the outbreak, we successfully isolated these two main clones from a radiant warmer and from nurses' hands. The radiant warmer and the nurses' hands may have played an important role in harboring the $K$. pneumoniae producing KPC-2 or NDM-1 carbapenemases that caused persistent infection within the same ward. Following the emergence of CRE on a ward, Screening and infection control measures must be implemented to block nosocomial transmission. Active and effective infection control measures are indispensable for preventing a subsequent outbreak.

\section{Additional files}

Additional file 1: Sequencing for resistance genes. (DOCX 17 kb)

Additional file 2: Sequencing for MLST. (DOCX $21 \mathrm{~kb}$ )

Additional file 3: Raw data of minimal inhibitory concentration. (XLSX $13 \mathrm{~kb}$ )

\section{Abbreviations}

CRE: Carbapenem -resistant Enterobacteriaceae; CR-KP: Carbapenem resistance -Klebsiella pneumoniae; ESBLs: Extended-spectrum beta-lactamases; KPC-2: K. pneumoniae carbapenemase; MIC: Minimal inhibitory concentration; MLST: Multilocus sequence typing; NDM-1: New Delhi metallo- $\beta$-lactamase-1 enzymes; ST: Sequencing type

\section{Funding}

This work was supported by the Infectious Diseases Control Project from Ministry of Health of China (2016zx10004-207). The funders had no role in study design, data collection and analysis, decision to publish, or preparation of the manuscript.

\section{Availability of data and materials}

All the data on which the conclusions of the manuscript rely is presented in the main paper and additional files 1, 2 and 3 .

\section{Authors' contributions}

$\mathrm{ZS}$ and LC designed the study; $\mathrm{YJ}$ and KT performed the experiments; ZR and YW collected the epidemiological data; ZC and XZ collected the microbiological data; LW, LT and WX analyzed the data; YJ wrote the manuscript. All authors read and approved the final manuscript.

\section{Competing interests}

The authors declare that they have no Competing interests.

\section{Consent to publish}

Not applicable.

\section{Ethics and consent to participate}

The study protocol was approved by the Tongji Hospital ethics committee for research in health. Informed written consent was obtained from the parents or legal guardian of the patient before inclusion in the study.

\section{Author details}

'Department of Clinical Laboratory, Tongji Hospital, Tongji Medical College Huazhong University of Science and Technology, Jiefang Road 1095\#, Wuhan 430000, China. ${ }^{2}$ Department of Infection Control, Tongji Hospital, Tongji Medical College Huazhong University of Science and Technology, Jiefang Road 1095\#, Wuhan 430000, China. ${ }^{3}$ Department of Pediatrics, Tongji Hospital, Tongji Medical College, Huazhong University of Science and Technology, Jiefang Road 1095\#, Wuhan 430000, China.

Received: 14 June 2016 Accepted: 27 September 2016

Published online: 12 October 2016

References

1. Patel G, Huprikar S, Factor Stephanie H, Jenkins Stephen G, Calfee David P. Outcomes of carbapenem-resistant Klebsiella pneumoniae infection and the impact of antimicrobial and adjunctive therapies. Infect Control Hosp Epidemiol. 2008;29(12):1099-106.

2. Grundmann H, Livermore DM, Giske CG, Canton R, Rossolini GM, Campos J, Vatopoulos A, Gniadkowski M, Toth A, Pfeifer Y, et al. Carbapenem-nonsusceptible Enterobacteriaceae in Europe: conclusions from a meeting of 
national experts. Euro surveillance. 2010;15(46). Available online: http://www. eurosurveillance.org/ViewArticle.aspx? Articleld=19711.

3. Doi Y, Paterson DL. Carbapenemase-producing Enterobacteriaceae. Semin Respir Crit Care Med. 2015:36(1):74-84.

4. Seiffert SN, Marschall J, Perreten V, Carattoli A, Furrer H, Endimiani A. Emergence of Klebsiella pneumoniae co-producing NDM-1, OXA-48, CTX-M-15, CMY-16, QnrA and ArmA in Switzerland. Int J Antimicrob Agents. 2014;44(3):260-2.

5. Leitner E, Zarfel G, Luxner J, Herzog K, Pekard-Amenitsch S, Hoenigl M, Valentin T, Feierl G, Grisold A, Hogenauer C, et al. Contaminated handwashing sinks as the source of a clonal outbreak of KPC-2-producing Klebsiella oxytoca on a hematology ward. Antimicrob Agents Chemother. 2015;59(1):714-6.

6. Glupczynski Y, Huang TD, Bouchahrouf W, Rezende de Castro R, Bauraing C, Gerard M, Verbruggen AM, Deplano A, Denis O, Bogaerts P. Rapid emergence and spread of OXA-48-producing carbapenem-resistant Enterobacteriaceae isolates in Belgian hospitals. Int J Antimicrob Agents. 2012;39(2):168-72.

7. Poirel L, Yilmaz M, Istanbullu A, Arslan F, Mert A, Bernabeu S, Nordmann P. Spread of NDM-1-producing Enterobacteriaceae in a neonatal intensive care unit in Istanbul, Turkey. Antimicrob Agents Chemother. 2014;58(5):2929-33.

8. Zhu J, Sun L, Ding B, Yang Y, Xu X, Liu W, Zhu D, Yang F, Zhang H, Hu F. Outbreak of NDM-1-producing Klebsiella pneumoniae ST76 and ST37 isolates in neonates. European journal of clinical microbiology \& infectious diseases: official publication of the European Society of Clinical Microbiology 2016; 35(4):611-8

9. Chen Z, Li H, Feng J, Li Y, Chen X, Guo X, Chen W, Wang L, Lin L, Yang H, et al. NDM-1 encoded by a pNDM-BJ01-like plasmid P3SP-NDM in clinical Enterobacter aerogenes. Front Microbiol. 2015;6:294. http://journal.frontiersin. org/article/10.3389/fmicb.2015.00294/full\#h8.

10. Papadimitriou M, Voulgari E, Ranellou K, Koemtzidou E, Lebessi E, Tsakris A. Emergence of VIM-1 metallo-beta-lactamase-producing Escherichia coli in a neonatal intensive care unit. Microb Drug Resist. 2011;17(1):105-8.

11. Lim KT, Yasin R, Yeo CC, Puthucheary S, Thong KL. Characterization of multidrug resistant ESBL-producing Escherichia coli isolates from hospitals in Malaysia. J Biomed Biotechnol. 2009;2009:165637.

12. Dallenne C, Da Costa A, Decre D, Favier C, Arlet G. Development of a set of multiplex PCR assays for the detection of genes encoding important beta-lactamases in Enterobacteriaceae. J Antimicrob Chemother. 2010;65(3):490-5.

13. Yang J, Ye L, Guo L, Zhao Q, Chen R, Luo Y, Chen Y, Tian S, Zhao J, Shen D, et al. A nosocomial outbreak of KPC-2-producing Klebsiella pneumoniae in a Chinese hospital: dissemination of ST11 and emergence of ST37, ST392 and ST395. Clin Microbiol Infect. 2013;19(11):E509-15.

14. Kallen A, Guh A. United States Centers for Disease Control and Prevention issue updated guidance for tackling carbapenem-resistant enterobacteriaceae. Euro Surveill. 2012;17(26). Available online: http://www. eurosurveillance.org/NiewArticle.aspx?Articleld=20207.

15. Liu C, Qin S, Xu H, Xu L, Zhao D, Liu X, Lang S, Feng X, Liu HM. New Delhi Metallo-beta-Lactamase 1(NDM-1), the Dominant Carbapenemase Detected in Carbapenem-Resistant Enterobacter cloacae from Henan Province, China. PLoS One. 2015;10(8):e0135044.

16. Borer A, Saidel-Odes L, Riesenberg K, Eskira S, Peled N, Nativ R, Schlaeffer F, Sherf M. Attributable mortality rate for carbapenem-resistant Klebsiella pneumoniae bacteremia. Infect Control Hosp Epidemiol. 2009;30(10):972-6.

17. Gómez Rueda V, Zuleta Tobón J J. Risk factors for infection with carbapenem-resistant Klebsiella pneumoniae: a case-case-control study[]]. Colombia Médica, 2014;45(2):54-60.

18. Jiao Y, Qin Y, Liu J, Li Q, Dong Y, Shang Y, Huang Y, Liu R. Risk factors for carbapenem-resistant Klebsiella pneumoniae infection/colonization and predictors of mortality: a retrospective study. Pathogens and Global Health. 2015;109(2):68-74.

19. Ulu-Kilic A, Alp E, Percin D, Cevahir F, Altay-Kurkcu C, Ozturk A, Ozturk A. Risk factors for carbapenem resistant Klebsiella pneumoniae rectal colonization in pediatric units. J Infect Dev Ctries. 2014;8(10):1361-4.

20. Lubbert C, Lippmann N, Busch T, Kaisers UX, Ducomble T, Eckmanns T, Rodloff AC. Long-term carriage of Klebsiella pneumoniae carbapenemase-2 producing K pneumoniae after a large single-center outbreak in Germany. Am J Infect Control. 2014:42(4):376-80.

21. Snitkin ES, Zelazny AM, Thomas PJ, Stock F, Henderson DK, Palmore TN, Segre JA. Tracking a hospital outbreak of carbapenem-resistant Klebsiella pneumoniae with whole-genome sequencing. Sci Transl Med. 2012:4(148):148ra116-148ra116.

\section{Submit your next manuscript to BioMed Central and we will help you at every step:}

- We accept pre-submission inquiries

- Our selector tool helps you to find the most relevant journal

- We provide round the clock customer support

- Convenient online submission

- Thorough peer review

- Inclusion in PubMed and all major indexing services

- Maximum visibility for your research

Submit your manuscript at www.biomedcentral.com/submit
Biomed Central 